\title{
Aéroélasticité : aspect numérique
}

\author{
Aeroelasticity : numerical aspect \\ par T. Fanion, \\ Dassault Aviation \& INRIA \\ G. Rogé, \\ Dassault Aviation
}

Aerodynamic unsteady phenomena, such as flutter, are modelized using robust conception tools. Although crucial for aircraft design, these low design tools are not accurate in the transonic domain. The usual approach (see [1] and [2]) relies on linear potential methods, calibrated with flight test measurements, wind tunnel experiments and sophisticated CFD codes simulations. Large improvements in both numerical methods and computers allow high levels of modelization (Navier-Stokes equations, ...). At the present time, we can consider computations which were formerly unreachable (see for example [11] and [7]). Focusing on accurate methods, we present in this paper results obtained with several modelizations of unsteady phenomena, each of them corresponding to precise needs (flight manoeuvre, small displacements of control surfaces, parafoil, ...). Finally, an alternate technique is currently being developped, halfway between classical tools and the most detailed modelizations, and begins being used in industrial context. The crucial point of this technique is to solve the linearized Euler (or Navier-Stokes) equations [3]). Results obtained using a linearized Euler solver (based on a discrete approach) are presented.

\section{I — MÉTHODES CLASSIQUES}

L'étude aéroélastique d'un avion représente une lourde charge de travail. L'ensemble des configurations de vol à étudier (quelques milliers, on doit en effet balayer tout un domaine de vitesses, accélérations, altitudes, configuration de masses et d'emports) est actuellement parcouru en utilisant la technique suivante :

- Calcul des déplacements du modèle structural pour quelques centaines de chargements (répartitions de pressions aérodynamiques) de base,

- Construction du modèle aérodynamique permettant d'exprimer les charges aérodynamiques stationnaires et instationnaires dans la base de charges précédente, en fonction du mouvement de l'avion et de ses gouvernes. Les effets des déformations "souples" stationnaires et les effets instationnaires (calculés en fréquentiel) sont obtenus par une méthode de singularités utilisant des "doublets" et des "sources".

- Grâce aux opérateurs précédemment construits, l'analyse du couplage aéroélastique peut alors être effectuée pour un coût dérisoire (voir [1]).

On montre sur la figure 1 un diagramme de flutter standard obtenu par le logiciel ELFINI de calcul de mécanique des structures de Dassault Aviation. Celui-ci utilise une méthode de "balayage en fréquence réduite" (voir [2]) pour la recherche de flottements. Sur le haut du diagramme on peut suivre l'évolution des fréquences des modes structuraux "mouillés", et sur le bas on suit les amortissements de ces modes. C'est ce type d'analyse qui sert (pour l'étude du flottement) de manière courante pour la conception et la certification des aéronefs.

\section{MÉTHODES À BASE DE CFD NON LINÉAIRE}

La priorité étant à l'analyse du domaine transsonique, domaine dans lequel les méthodes de singularités ont un comportement très discutable (qui impose de nombreux recalages), dans le cas de grands badins (et donc d'incidences modérées !), la modélisation du fluide est composée des équations d'Euler, couplées, si nécessaire, à des "équations de couche-limite".

Le code Euler utilisé (code EUGENIE de Dassault Aviation) est basé sur une formulation conservative, de type Volumes Finis, en maillages non structurés (Finite Volume Cell Vertex, voir [4]). Les schémas utilisés dans le domaine transsonique sont du type centré.

Pour le couplage, le comportement dynamique de la structure est condensé par la construction d'un ensemble d'opérateurs linéaires fournis par ELFINI, permettant de calculer l'évolution du déplacement des nœuds du maillage aérodyna- 


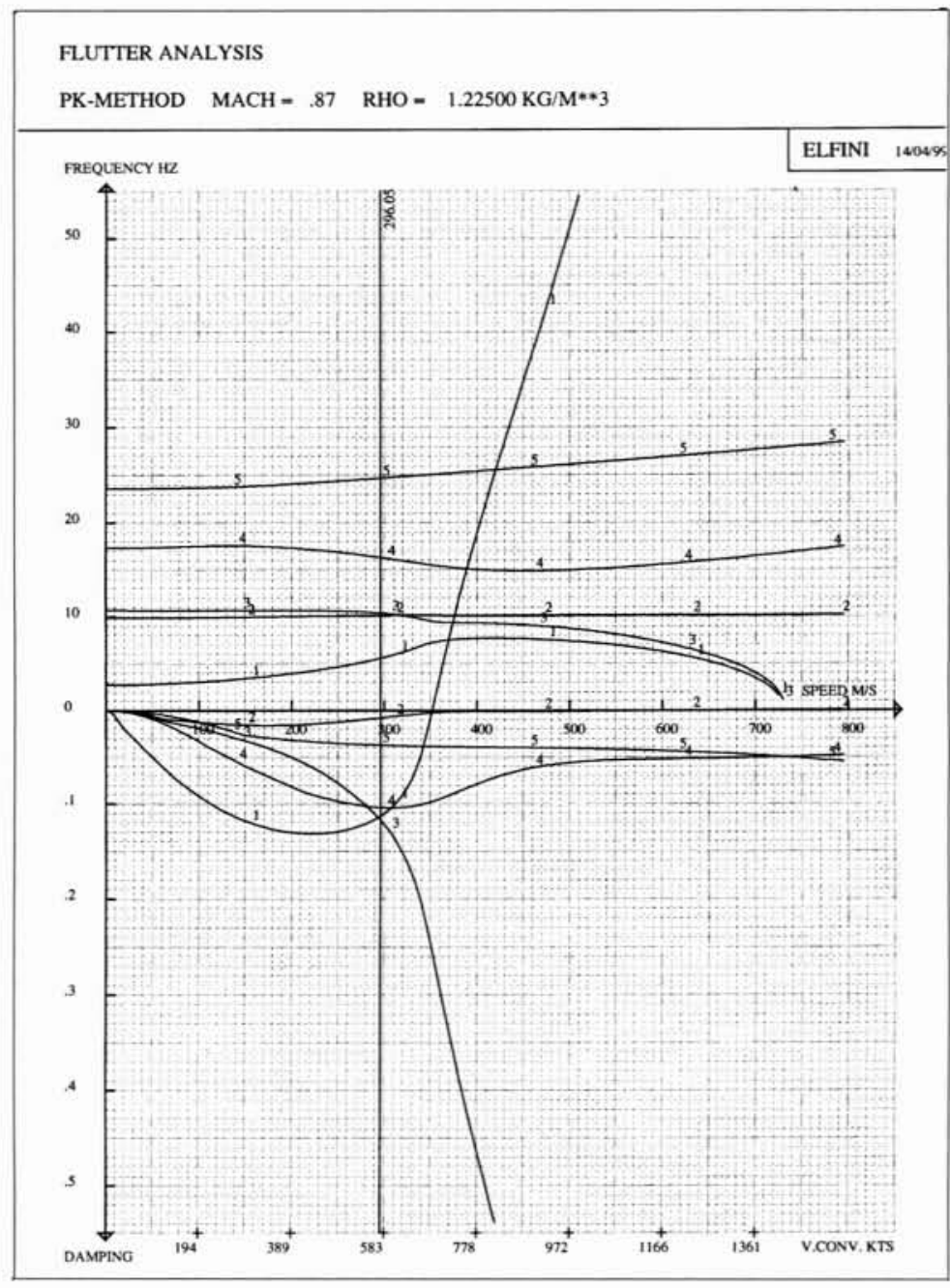

1. Diagramme de flutter ELFINI, aile de type civil.

mique à la paroi de l'avion en fonction des pressions aux facettes de la paroi du maillage CFD. Pour les problèmes de transferts d'informations entre les grilles des problèmes aérodynamique et structural ("matcher"), on consultera par exemple [10].
Des déformées, correspondant aux premiers modes structuraux dans le vide, sont présentées sur les figures 2 (dérive du Rafale) et 3 (aile de Mirage F1).

Les déplacements à la paroi sont pris en compte, dans le modèle Euler, par une condition dite de transpiration (voir [12]) ou par une technique de maillages dynamiques (voir [5]).

Un organigramme (figure 4) présente la chaîne instationnaire dans le cas de "maillages dynamiques".

Des algorithmes à temps décalés plus sophistiqués peuvent également être mis en œuvre (voir [8]).

Pour un calcul transsonique d'oscillations de tangage d'une aile (construite à partir du profil NACA $64 \mathrm{A010}$ ), on présente sur les figures 5 et 6 une comparaison des premières harmoniques des coefficients de pression pour les trois approches suivantes :

- méthode de transpiration (ou encore de paroi mobile),

- méthodes de type A.L.E. (maillages dynamiques),

- calcul en repère non-galiléen.

On notera que l'approche "maillages dynamiques" (avec GCL ordre 2, voir [9]) donne des résultats tout à fait comparables aux autres, que ce soit avec un maillage déformé (laplacien par composante du déplacement), ou avec un maillage subissant une rotation d'ensemble. Sur les figures 7 et 8 , on montre une superposition de ces deux maillages (dans une coupe $\mathrm{y}=\mathrm{cst}$ ) pour une rotation de dix degrés.

Pour illustrer le couplage fluide-structure, on présente, sur la figure 9, l'évolution de l'énergie structurale totale (énergie cinétique + énergie potentielle) au cours du temps dans un cas sans flutter et dans un cas avec flutter (irréaliste) de la dérive du Rafale (figure 10).

Une analyse de type Fourier (par exemple trois périodes ont été nécessaires pour obtenir une solution périodique) ou

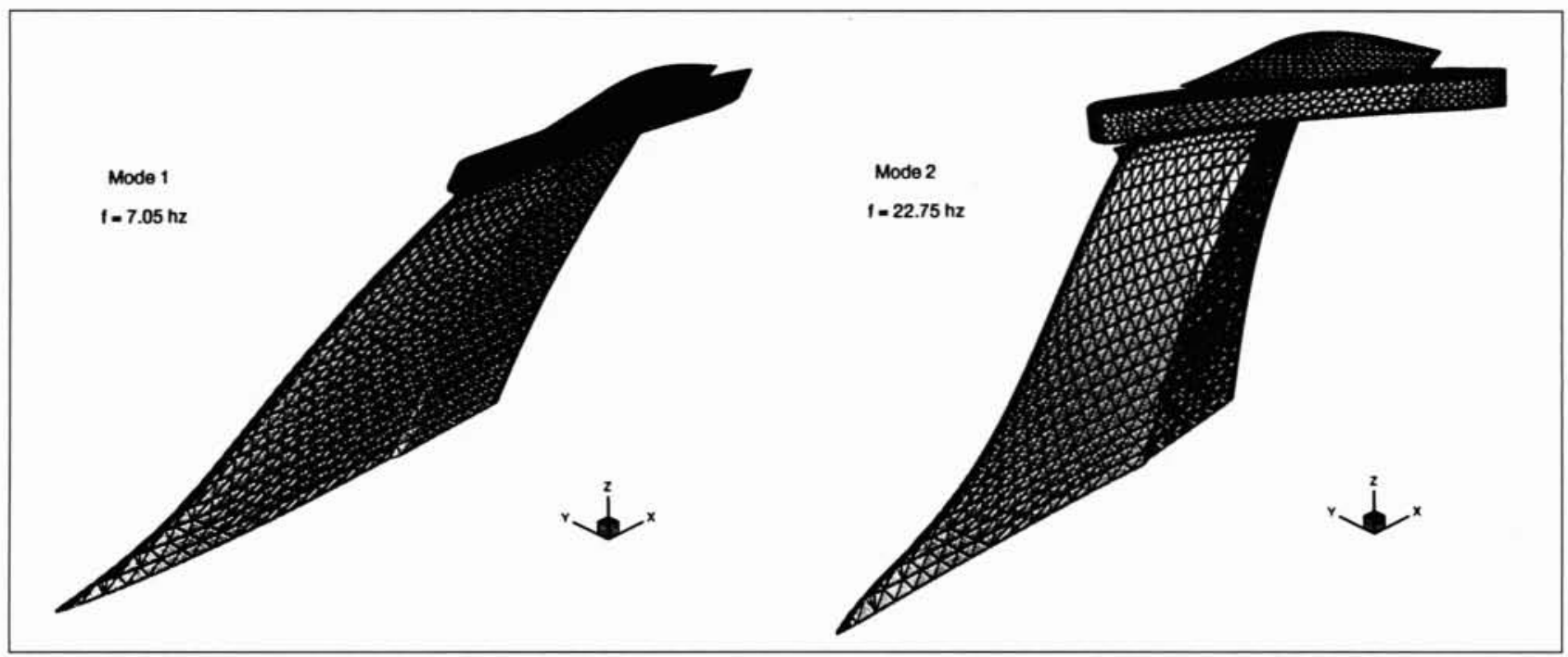

2. Premiers modes structuraux dans le vide pour la dérive du Rafale. 

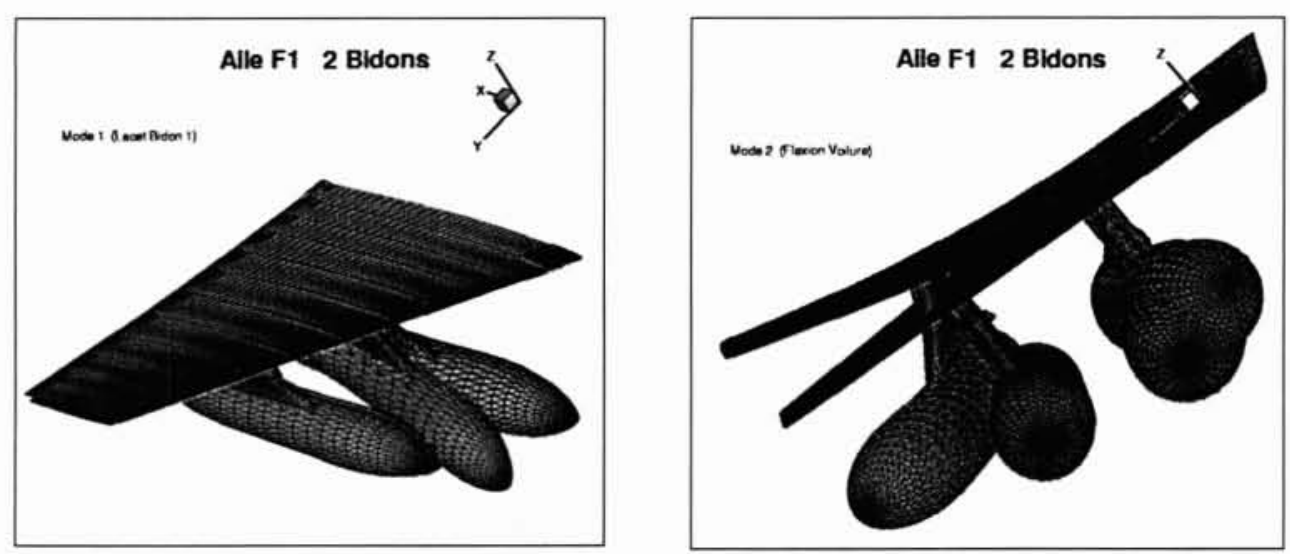

3. Premiers modes structuraux dans le vide pour l'aile de Mirage F1.
Prony (consulter [13]) doit être effectuée pour obtenir l'information recherchée. Dans le cas d'une évolution temporelle d'un paramètre (comme le nombre de Mach, dans le cas d'un avion en accélération), les fréquences et les amortissements des modes structuraux sont dépendants du temps ; il faut alors utiliser des méthodes de traitement du signal plus sophistiquées (voir [14]) que l'analyse de Prony.

Chez SAAB, les responsables du diagnostic de "flutter jugent" (Projet européen UNSI, Minutes of 18-Month Meeting at IMFT in Toulouse 5/6 July 1999) qu'un remplacement de la méthode présentée dans le chapitre I n'est pas envigeable à court et moyen termes. En effet, une analyse de flutter pour 100 configurations de charge leur coûte 5 heures sur une SGI R10000 avec cette méthode, alors qu'un simple calcul Navier-Stokes, sur une aile (600 000 sommets), demande plus de 400 heures sur 4 processeurs SGI R10000.

\section{MÉTHODES À BASE DE CFD LINÉARISÉE}

Un bon compromis entre les méthodes présentées en I et II réside dans l'utilisation d'une CFD linéarisée. Cette approche suppose l'existence, autour de l'état d'équilibre sta- tique étudié, d'un domaine où les équations CFD sont différentiables.

Deux voies sont alors possibles :

- Le calcul des réponses à des petites perturbations stationnaires ou harmoniques (pour une base de déformées et un ensemble de fréquences, ou encore pour des perturbations sur des paramètres tels que le nombre de Mach, la vitesse de tangage, ou encore l'angle d'incidence). Les FAG (Forces Aérodynamiques Généralisées) obtenues se substituent alors naturellement à celles initialement calculées par la méthode des doublets. On peut ensuite appliquer la méthode dite du "balayage en fréquence réduite", comme dans la partie I, pour résoudre les équations du flottement.

- La résolution du problème aux valeurs propres complexes obtenu par linéarisation des équations d'équilibre du problème aéroélastique.

La première approche a été adoptée par l'ONERA (voir [15]), avec deux variantes : la transpiration et la déformation de maillage. La seconde approche est décrite dans [6]. Les auteurs y proposent une méthode des puissances itérées inverses et orthogonales et se placent dans une formulation à trois champs : le fluide, son mouvement de maillage et la structure.

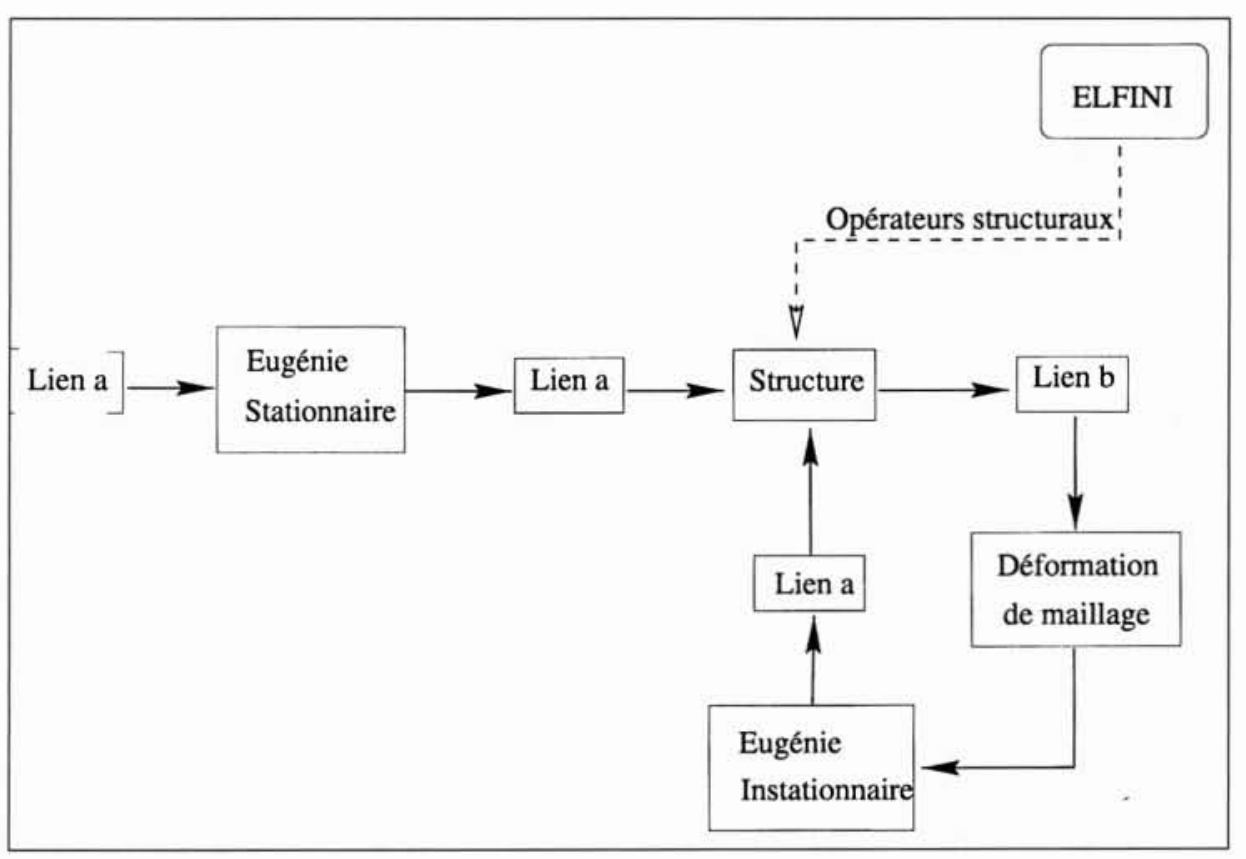

4. Chaîne

d'aéroservoélasticité. 


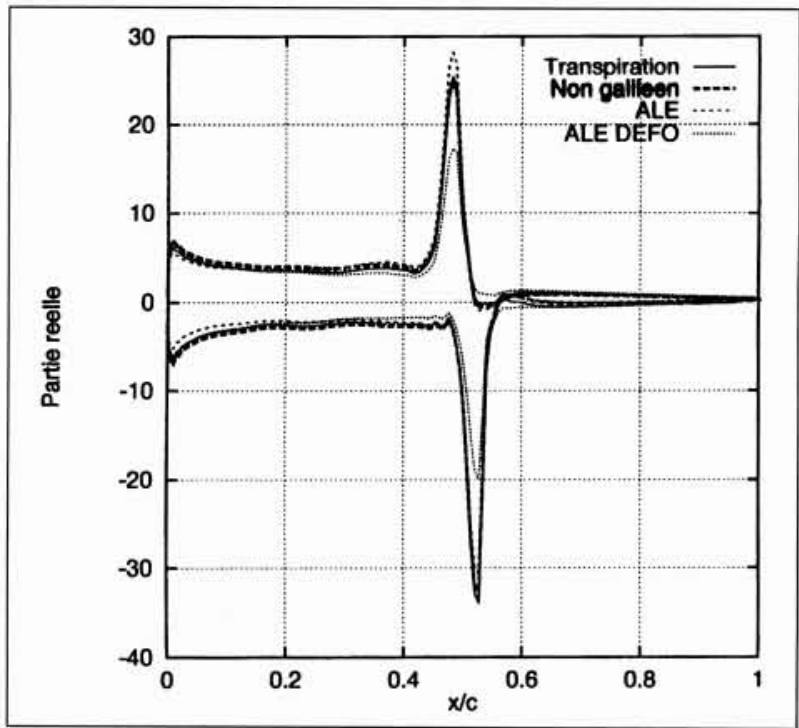

5. Partie réelle (première harmonique du coefficient de pression).

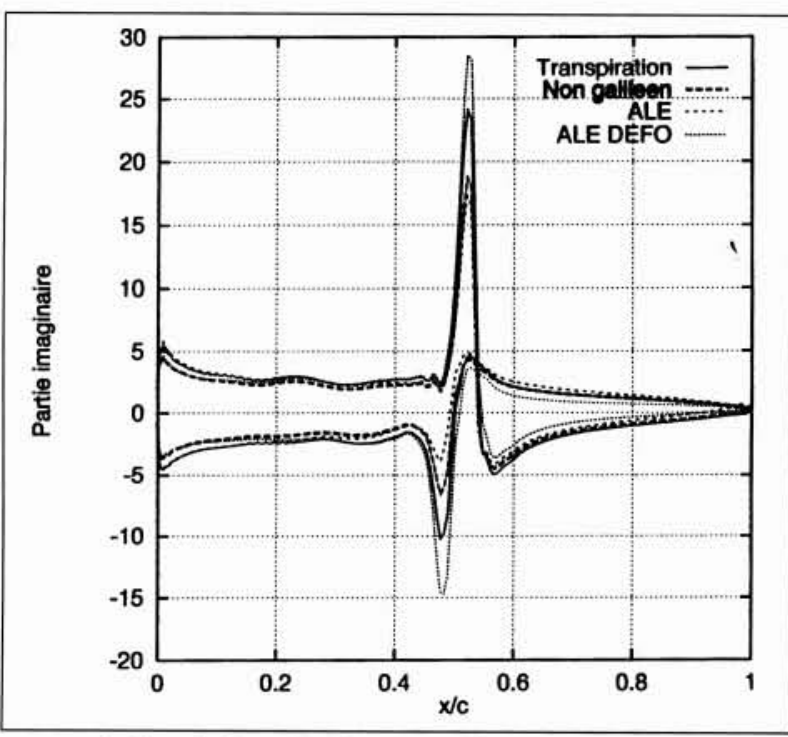

6. Partie imaginaire (première harmonique du coefficient de pression).

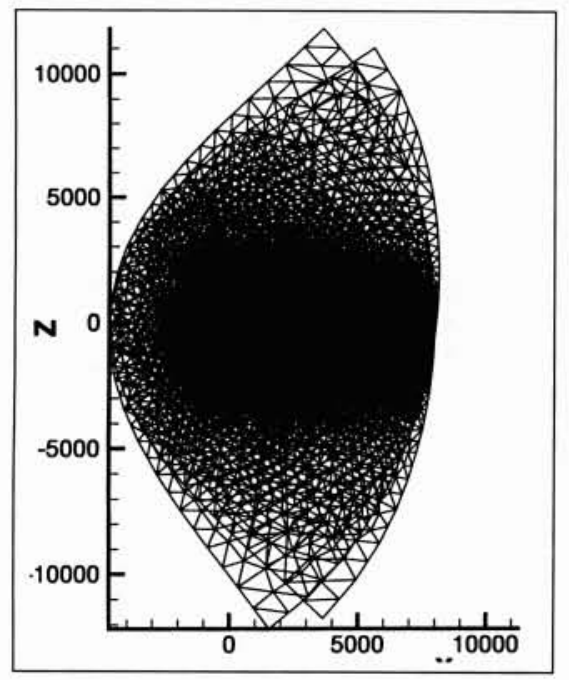

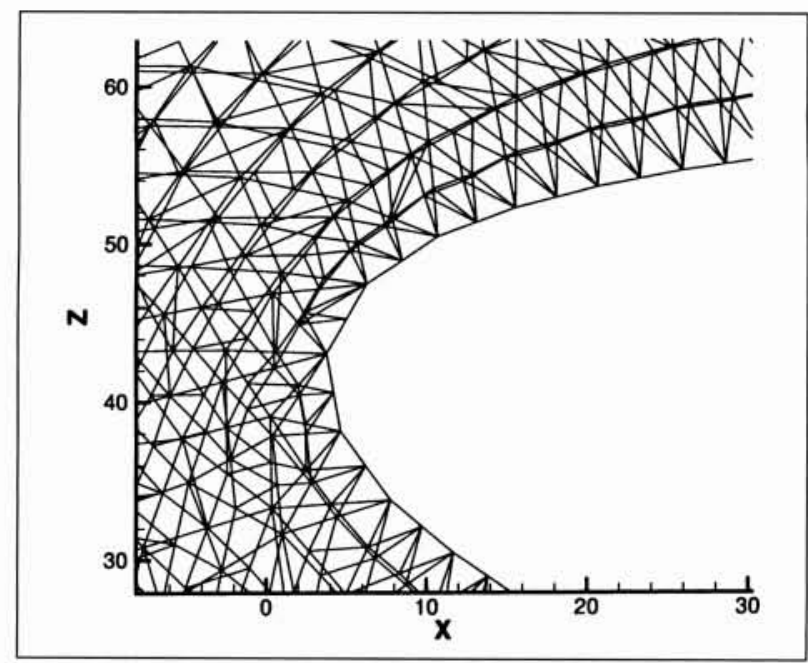

8. Vue partielle des deux maillages obtenus après $10^{\circ}$ de rotation.

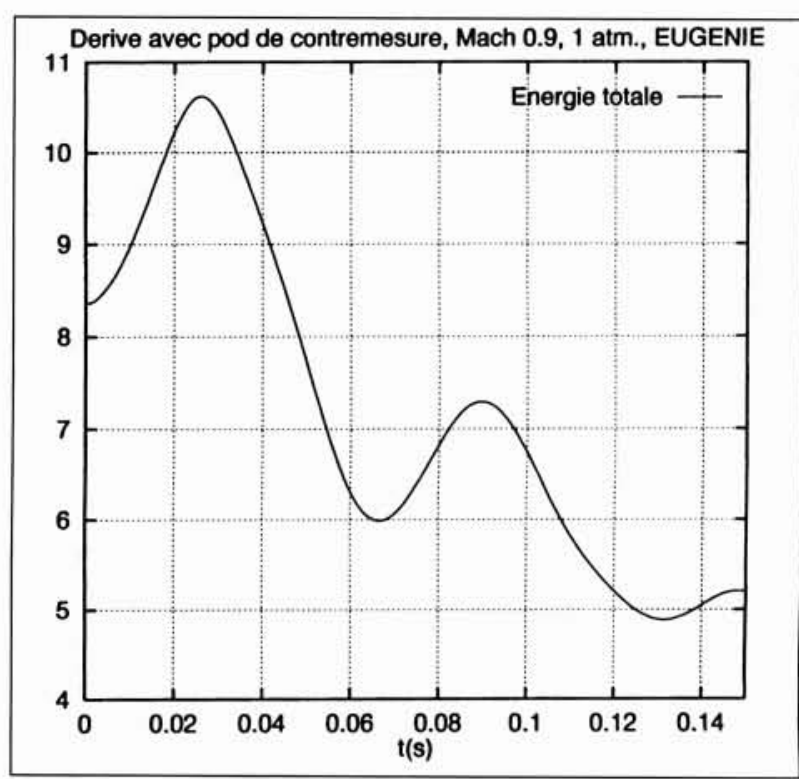

9. Dérive du Rafale, Energie totale, cas sans flutter.

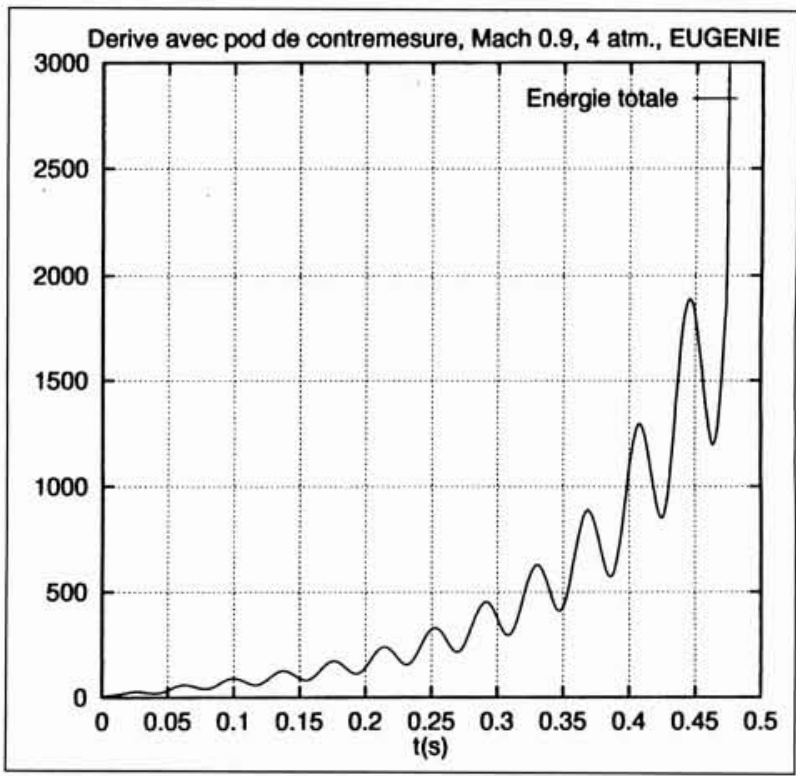

10. Dérive du Rafale, Energie totale, cas avec flutter. 


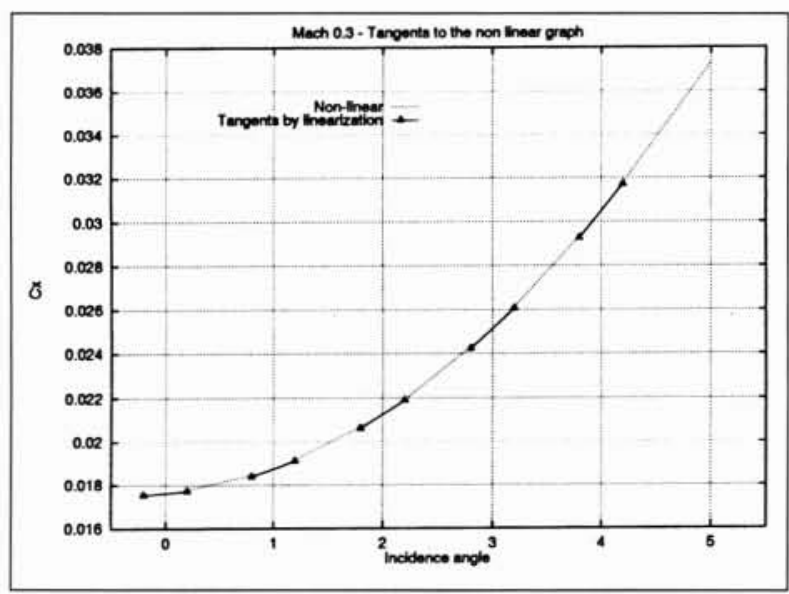

11. Pentes de la traînée en fonction de l'incidence à Mach 0,3.

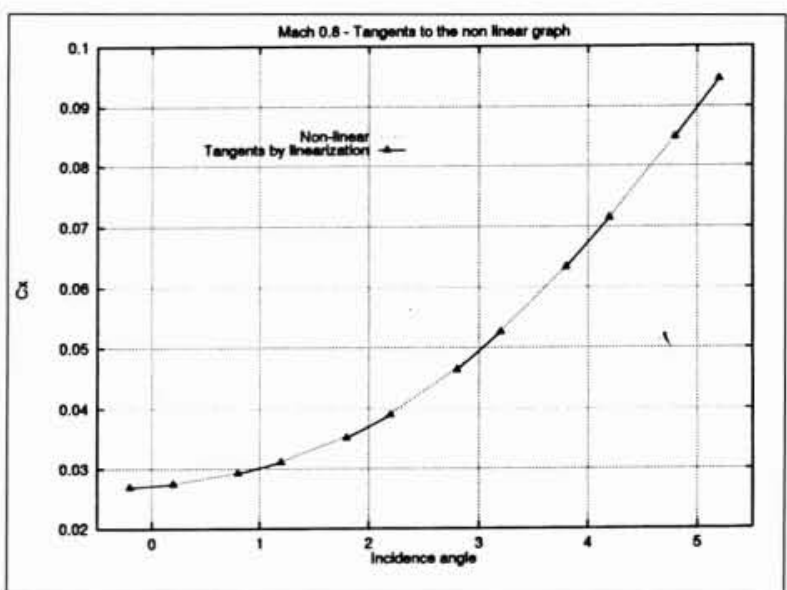

12. Pentes de la traînée en fonction de l'incidence à Mach 0,8.

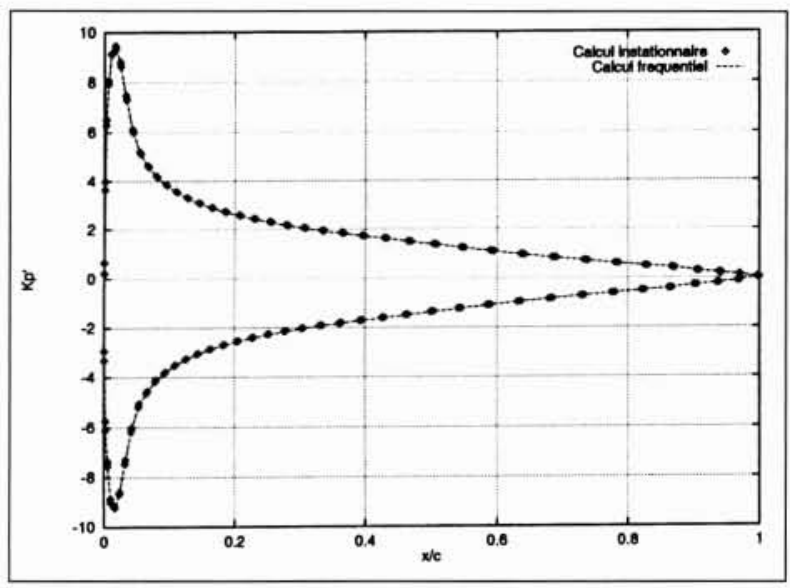

13. Parties réelles des coefficients de pression pour une oscillation de tangage.

L'intérêt pour un code Euler linéarisé s'est manifesté chez Dassault Aviation et a ammené à son développement au sein du logiciel EUGENIE. Des résultats obtenus sur une aile civile (ONERA M6) avec ce code Euler linéarisé stationnaire (autour d'un écoulement stationnaire) de Dassault Aviation sont présentés sur les figures 11 (subsonique, Mach $=0,3$ ) et 12 (transsonique, Mach $=0,8$ ).

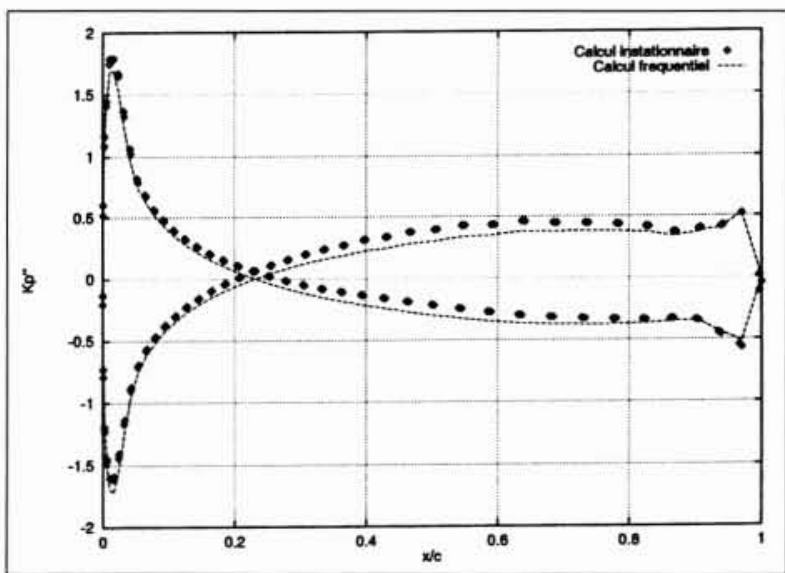

14. Parties imaginaires des coefficients de pression pour une oscillation de tangage.

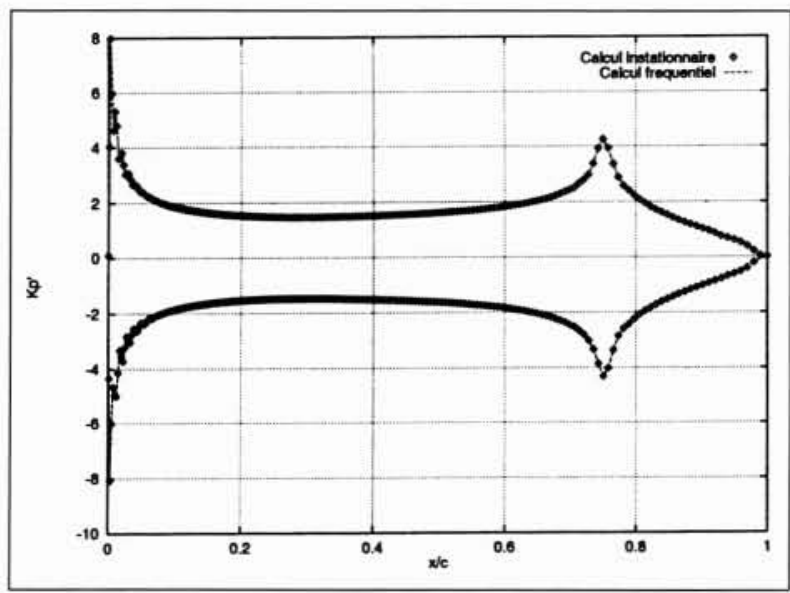

15. Parties réelles des coefficients de pression pour une oscillation de gouverne.

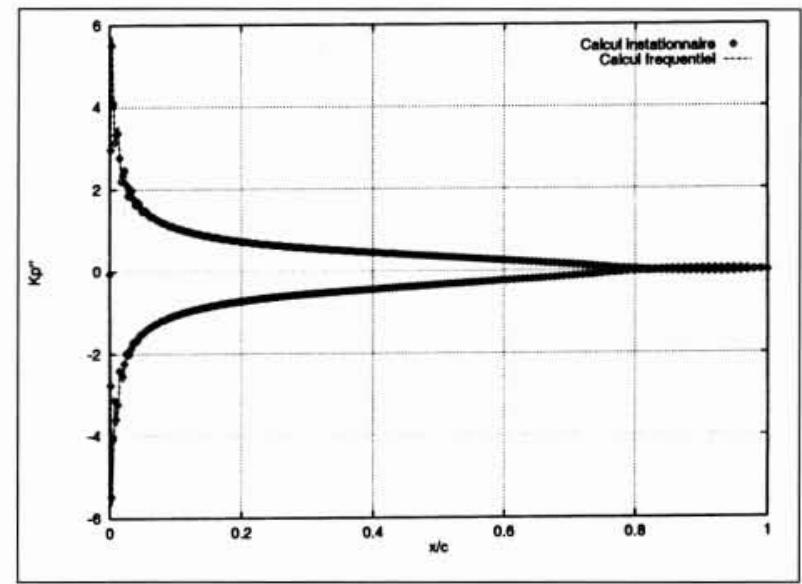

16. Parties imaginaires des coefficients de pression pour une oscillation de gouverne.

Une variante harmonique de ce code a également été développée ; elle permet de calculer directement des quantités comme la première harmonique du coefficient de pression. On présente une comparaison des résultats instationnaires et harmoniques obtenus en régime subsonique (Mach $=0,5)$ sur un exemple d'oscillations de tangage (aile ONERA M6, fréquence $=20 \mathrm{~Hz}$ ), sur les figures 13,14 , puis 
sur un exemple d'oscillation de gouverne (aile construite à partir du profil NACA $64 \mathrm{~A} 006$, fréquence $=5 \mathrm{~Hz}$ ) sur les figures 15 et 16 .

Le temps de calcul (écoulement stationnaire non compris) a été divisé par 12,5 par rapport au calcul instationnaire (trois périodes). Contrairement à l'approche adoptée dans [15], on n'utilise pas une technique de pas de temps dual pour le calcul linéarisé, ce qui nous permet d'envisager une résolution avec plusieurs seconds-membres à moindre coût.

\section{VI $\square$ CONCLUSION}

Après de nombreuses années pendant lesquelles seule la méthode à base de potentiel linéaire présentée dans la partie I était utilisable lors de la conception et la certification des aéronefs, de nouvelles approches émergent enfin. Historiquement, les mécaniciens des fluides se sont lancés dans une modélisation fine (couplage CFD non linéaire - Modèle structural général, en domaine temporel). Dans ce domaine, les avancées ont été nombreuses et la stratégie de couplage (voir [8]) est maintenant bien maîtrisée.

Cependant, le coût CPU limite l'utilisation de ces approches à quelques points critiques, pour lesquels on doute de la validité des hypothèses simplificatrices effectuées. A court et moyen termes, la stratégie à adopter pour améliorer le diagnostic de flutter est claire : il faut, dans le domaine transsonique, remplacer la méthode des doublets par de la CFD linéarisée. Les ordinateurs actuels permettent en effet (calcul parallèle sur machine à mémoire distribuée, ... ) d'obtenir par cette technique des temps CPU très raisonnables.

\section{Remerciements}

Nous remercions Svetlana Bozinoski, Charbel Fahrat, Guy Mortchéléwicz et Christian Petiau pour les conversations fructueuses que nous avons eues.

\section{RÉFÉRENCES}

[1] C. Petiau, S. Brun, Tendances actuelles de l'analyse aéroélastique des avions militaires, conférence AGARD, proceeding $n^{\circ} 403$. Athènes, 1986

[2] R. Dat, JL. Meurzec, Sur les calculs de flottement par la méthode dite du "balayage en fréquence réduite", La Recherche Aérospatiale, $\mathrm{n}^{\circ}$ 133, 1969.
[3] Q. V. Dinh, T. Fanion, G. Rogé, C. Sevin, A discrete approach for the calculation of aerodynamic sensibilities, sixteenth International Conference on Numerical Methods in Fluid Dynamics, Arcachon, 1998.

[4] L. Fezou, B. Stoufflet, A class of implicit upwind schemes for Euler simulations with unstructured meshes, Journal of Computational Physics 84:174-206, 1989 .

[5] S. PIPERNo, Simulation numérique des phénomènes d'interaction fluidestructure, Thèse de l'Ecole Nationale des Ponts et Chaussées, juin 1995.

[6] M. Lesoinne, C. FAhrat, Re-engineering of an Aeroelastic Code for Solving Eigen-Problems in all Flight Regimes, AIAA Paper 97-0647, 35th Aerospace Sciences Meeting and Exhibit, Reno, Nevada, January 6-9, 1997.

[7] DJ. Rixen, C. Fahrat, LD. Peterson, Simulation of The Continuous Parametric Identification of an Accelerating Aeroelastic System, AIAA Paper 99-0797, 37th Aerospace Sciences Meeting and Exhibit, Reno, Nevada, January 11-14, 1999.

[8] C. FAhrat, M. LesolnNe, Higher-Order Staggered and Subiteration Free Algorithm for Coupled Dynamic Aeroelasticity Problems, AIAA Paper 98-0516, 36th Aerospace Sciences Meeting and Exhibit, Reno, Nevada, January 12-15, 1998.

[9] B. Koobus, C. Fahrat, Second-order implicit schemes that satisfy the GCL for flow computations on dynamic grids, AIAA Paper 98-0113. 36th Aerospace Sciences Meeting and Exhibit, Reno, Nevada, January 12-15, 1998

[10] C. FAhrat, M. Lesoinne, P. Le TAllec, Load and motion transfer algorithms for fluid/structure interaction problems with non-matching discrete interfaces: Momentum and energy conservation, optimal discretization and application to aeroelasticity. Comput. Meths. Appl. Mech. Engrg., 157:95-114, 1998.

[11] F. Сhalot, JP. Tribot, M. Rapuc, G. Durand, Large gliding parachute: experimental and theoretical approaches. AIAA Paper 97-1482, 14th Aerodynamic Decelerator Systems Technology Conference, San Francisco, California, June 2-5, 1997.

[12] MJ. LightriLL., On displacement thickness. Journal of Fluid Mechanics. vol. 4, pp. 383-392, 1958

[13] GRB. Prony, Essai expérimental et analytique, Journal de l'Ecole Polytechnique, 1, cahier 2, 1795 .

[14] P. Duvaut, F. Dublanchet, P. Lemoine, Sintrack, un algorithme de détection et d'estimation adaptatives de sinusoïdes exponentiellement amorties et décalées dans le temps. Quatorzième Colloque Gretsi, Juanles-Pins, septembre 1993.

[15] GD. Mortchéléwicz, Application des équations d'Euler linéarisées à la prévision du flottement, 85th AGARD SMP meeting, Aalborg, Danemark, 13-16 novembre 1997. 